Cahiers $d u$ MONDE RUSSE

\section{Cahiers du monde russe}

Russie - Empire russe - Union soviétique et États indépendants

$57 / 4 \mid 2016$

Varia

\title{
Arkadi Zeltser, éd., To pour out my bitter soul. Letters of Jews from the USSR 1941-1945
}

\section{Boris Czerny}

\section{OpenEdition}

\section{Journals}

Édition électronique

URL : http://journals.openedition.org/monderusse/10047

DOI : $10.4000 /$ monderusse. 10047

ISSN : $1777-5388$

\section{Éditeur}

Éditions de l'EHESS

\section{Édition imprimée}

Date de publication : 1 octobre 2016

Pagination : 979-982

ISBN : 978-2-7132-2542-0

ISSN : $1252-6576$

Référence électronique

Boris Czerny, «Arkadi Zeltser, éd., To pour out my bitter soul. Letters of Jews from the USSR

1941-1945 », Cahiers du monde russe [En ligne], 57/4 | 2016, mis en ligne le 01 octobre 2016, Consulté le 25 septembre 2020. URL : http://journals.openedition.org/monderusse/10047 ; DOI : https:// doi.org/10.4000/monderusse. 10047 
11 - Lev Bakst, Moja duša otkryta, t. 2, p. 12.

12 - Léon Bakst, Correspondance et morceaux choisis, traduction et présentation de Jean-Louis Barsacq, P. : L'Âge d'homme, 2016, p. 32.

13 - Ibid., p. 167.

\section{Olga Medvedkova \\ CNRS-ENS}

\section{Arkadi ZELTSER, éd.}

\section{To pour out my bitter soul Letters of Jews from the USSR 1941-1945}

Jérusalem : Yad Vashem, 2016, bibliographie, index, 276 p.

En dépit des travaux publiés récemment sur les massacres de masse en URSS durant la Seconde Guerre mondiale ${ }^{1}$, en dépit également des recueils passionnants sur ce sujet édités régulièrement par l'organisation russe « Centr i Fond "Holokost" »" de nombreux aspects de la vie quotidienne de la population et des territoires occupés pendant le conflit, et tout particulièrement des Juifs de Russie, restent à ce jour peu ou mal connus. Cette lacune est partiellement comblée par le présent ouvrage édité par le Mémorial de Yad Vashem. Les cent lettres qu'il contient sont réparties thématiquement dans sept chapitres intitulés « Dernières lettres », «Premières réactions aux meurtres de masse », « Réactions à l'antisémitisme », «Vie juive à l'arrière », «Vie juive dans les territoires libérés », «Le patriotisme soviétique et l'image du soldat juif », «Le destin des unités militaires juives dans l'Armée rouge ». À l'intérieur de chaque partie, les lettres, traduites en anglais du russe et du yiddish et dont il aurait été passionnant de disposer des textes originaux, sont classées chronologiquement. L'ensemble est précédé d'une introduction précise et stimulante rédigée par A. Zeltser. Ces cent lettres proviennent du fonds Yad Vashem et du fonds du Comité juif antifasciste (aux Archives d'État de la Fédération de Russie, GARF), auxquels un nombre important de destinateurs juifs s'adressa tout au long de la guerre. Elles s'inscrivent dans la même approche « juive » de la guerre que celle qui avait prévalu il y a de cela quelques années pour la publication d'une série de livres consacrés à la correspondance entre des Juifs soviétiques et l'écrivain Il'ja Erenburg $^{3}$ et des lettres envoyées par des soldats soviétiques juifs à leurs familles depuis le front. Cependant, par rapport à ces publications, To pour out my bitter soul, qui comporte un nombre substantiel de lettres envoyées à Erenburg, apporte une nuance essentielle sur la judéité affirmée des auteurs, judéité qui s'exprime dans la « chair » même des lettres, que ce soit par des références aux fêtes juives et à la religion, l'utilisation imparfaite du russe ou l'emploi du yiddish. Outre les moyens linguistiques, comme le choix d'une langue juive, l'affirmation d'une fierté juive prend parfois des formes touchantes et naïves. C'est le cas dans un certain nombre de lettres dont les auteurs énumèrent les figures historiques du judaïsme universel. Ainsi, dans la lettre 87, le destinateur loue la grandeur du « peuple juif 
qui a donné au monde Marx et Heine, Sholem Haleichem, Einstein, Sverdlov et Kaganovič, Spinoza et I.L. Peretz ». Mais au-delà de ces listes hétérogènes et imparfaites, il faut surtout retenir la puissance de l'affirmation identitaire juive qui se fait sentir dans l'ensemble des correspondances réunies. De fait, le choix de la phrase « To pour out my bitter soul » comme titre général du livre, phrase tirée de la lettre 85 et qui fait manifestement écho au Livre des Lamentations ou Lamentations de Jérémie qui servit de référence biblique à l'interprétation des catastrophes frappant le monde juif tout au long de son histoire jusqu'à la Shoah, ne paraît pas forcément le plus judicieux. Certes, de nombreux auteurs manifestent leur douleur et leur peine d'avoir perdu des proches lors des massacres perpétrés par les nazis et, de même, des combattants traversant les territoires occupés par les Allemands, font, à l'instar de Vasilij Grossman, le terrible constat qu' « il n'y a plus un seul Juif vivant en Ukraine » (lettres 39 et 40), mais, dans leur ensemble, les destinateurs des lettres font preuve d'un très fort esprit combatif dans l'affirmation de leur identité, qu'elle soit retrouvée - en cela ils s'inspirent d'Erenburg affirmant dans un poème de 1941 que les Allemands lui avaient rappelé que sa mère s'appelait Hanna (lettres 14, 15) -, ou bien encore renforcée dans ses certitudes et ses exigences. De fait, le concept « d'ethnicité incertaine » élaboré par l'historien Zvi Gitelman au sujet des Juifs soviétiques ${ }^{4}$ semble bien mal adapté pour les auteurs des lettres. L'adoption de la doxa soviétique et de formules clichés sur « l'amitié entre les peuples » contamine le discours - au sens large - des auteurs, qui utilisent le calque du « grand peuple russe » pour parler du " grand peuple juif » (lettres 15 et 87). Mais c'est précisément au nom de cette grandeur juive que de nombreux correspondants soulignent l'absolue nécessité de se venger et réclament que soit affirmé bien haut en russe comme en yiddish, sur les scènes de théâtre et dans les journaux, l'héroïsme des soldats juifs combattant en tant que Juifs pour la survie de leur peuple et non au nom d'idéaux communistes. Les militaires engagés sur le front et les simples citoyens dont les lettres sont présentées dans le recueil ont presque tous conscience d'incarner le peuple, la nation juive dans son ensemble, de Moscou à Tel-Aviv, du Birobidžan à Brooklyn. Héroïques ${ }^{5}$ et fidèles au régime, même s'ils sont Juifs avant d'être Soviétiques, les auteurs des lettres ne cèdent pas au désespoir, mais, au contraire, pour la plupart, ils exposent avec force des exigences qu'ils estiment légitimes, comme la parution de journaux juifs en nombre plus important, la création d'opéras, et l'ouverture de théâtres (lettre 69). Un auteur demande même d'exproprier les Ukrainiens de certains territoires en raison de leur collaboration avec les Allemands et l'attribution de ces mêmes territoires à des communautés ou colonies juives pour les dédommager des pertes subies (lettre 49). Cette revendication émane d'un Juif non religieux, qui évoque les livres de prières qui attendent leurs propriétaires dans un shtetl de Pologne et qui, dans un geste spontané, se saisit d'une Hagada de Pessah. Il est par ailleurs à noter que dans leur immense majorité, les lettres proviennent de correspondants se trouvant dans des villes et localités situées à l'intérieur des frontières de l'URSS. Le dernier cas cité se rapportant à la Pologne est une exception notable.

La lecture de ces cent lettres permet de ressentir la Shoah de l'intérieur d'un point de vue essentiellement émotionnel. Les correspondances ne peuvent pas 
servir en tant que documents complémentaires à une histoire froide et raisonnée de la Shoah. Les témoins crient et hurlent. Ils s'adressent au monde entier pour que soit reconnu le massacre de six millions de Juifs. Très vite, et c'est même assez surprenant, les auteurs sont au fait des tueries perpétrées dans différentes villes et du fonctionnement des camps d'extermination. Les lettres apparaissent donc comme un médium parallèle ou complémentaire aux media officiels. Mais surtout, l'ensemble de ces correspondances dévoile un « monde » juif russe dont l'assimilation est moins avancée que ce qui est généralement affirmé. En dépit de l'afflux de nombreux Juifs dans les villes et de la disparition de tout un tissu culturel, religieux, associatif dans les années 1920 et 1930, les Juifs soviétiques, tels qu'ils apparaissent dans leur diversité à travers ces cent lettres, sont très fortement imprégnés d'un puissant sentiment positif d'appartenance identitaire, qui leur permet de considérer Erenburg et Mikhoels comme leurs uniques porte-parole officiels auprès du gouvernement soviétique.

Outre cette dimension nationale ou nationaliste qui émerge à la lecture de l'ouvrage, trois autres points attirent l'attention. Le premier concerne la puissance de l'antisémitisme en URSS aussi bien sur le front qu'à l'arrière et ce, dès la seconde moitié des années 1942 et surtout à partir du début de 1943 et de la victoire à Stalingrad. Cet aspect n'est pas une « découverte », mais jusqu'à présent l'appréhension de ce phénomène était limitée aux sphères officielles ${ }^{6}$. Pour reprendre une expression utilisée par un destinateur (lettre 49), « l'antisémitisme [en Russie et dans les territoires libérés] se sent à chaque pas ». Le deuxième point, évoqué par plusieurs correspondants, porte sur l'enrôlement dans les rangs de l'Armée rouge de personnes ayant collaboré avec les nazis. Ainsi l'auteur de la lettre 43, s'insurge contre la réhabilitation de fait, après la libération, d'anciens collaborateurs qui « maintenant jouent les premiers violons. Ceux qui criaient mort aux Youpins, ajoute-t-il, continuent maintenant de hurler des slogans antisémites sans aucune opposition ». À notre connaissance, il n'existe pas ou peu d'études sur ce sujet de l'épuration ou, a contrario, de l'absence volontaire d'épuration.

Enfin, le troisième et dernier aspect se rapporte au destin même des lettres qui, en plein conflit, furent expédiées très loin des lieux de massacres et réceptionnées en Palestine ou au Birobidžan, certaines très rapidement après leur envoi, d'autres bien des années plus tard. L'histoire de la transmission des informations en général et de celles portant sur la Shoah en temps de guerre apparaît également un champ de recherches à explorer.

Par sa densité et sa richesse, par l'émotion qu'il suscite à la lecture de chacune de ses lignes, le livre publié sous la direction d'Arkadi Seltzer ouvre des horizons bien plus vastes que celui auquel le nombre de cent, représentant les lettres réunies, ne semble devoir le restreindre.

1 - Annie Appelboin, Assia Kovriguina, dir., Témoigner sur la Shoah en URSS, Paris 16-17 mai 2013, http://www.fabula.org/colloques/sommaire2672.php ; Catherine Coquio, La Littérature en suspens. Écriture de la Shoah : le témoignage et les œuvres, P. : L'Arachnéen, 2015. 
2 - Centr i Fond Holokost [Centre et fonds de l'Holocauste] : http://www.holocf.ru/

3 - Mordechai Altshuler, Arad Yitzhak, Samuel Krakowski, éds., Sovetskie evrei pišut

Il'e Erenburgu [Les Juifs soviétiques écrivent à Il'ja Erenburg], Jérusalem : The Centre for Research and Documentation of East-european Jewry, The Hebrew University of Jerusalem, Yad Vashem, 1993 ; Il’ja Al’tman, Leonid Teruškin, éds., Sohrani moi pis'ma... Sbornik pisem i dnevnikov perioda Velikoj Otečestvennoj vojny [Conserve mes lettres... Recueil de lettres et de journaux intimes de la Grande Guerre patriotique], M. : Tsentr i fond 'Kholokost', MIK, 4 ouvrages de 2007 à 2016.

4 - Zvi Y. Gitelman, Jewish Identity in Post Communist Russia and Ukraine : An Uncertain Ethnicity, Cambridge : Cambridge University Press, 2012, p. 16-17.

5 - Enciklopedija evrejskogo geroizma [Encyclopédie de l'héroïsme juif], M. : Dograf, 2002 ; Mordechai Altshuler, « Jewish combatants of the Red Army Confront the Holocaust », in Harriet Murav, Gennady Estraikh, dir., Soviet Jews in World War II : Fighting, Witnessing, Remembering, Boston : Academic Studies Press, 2014, p. 16, 31-32.

6 - Genadij Kostyrčenko, Gosudarstvennyj antisemitizm $v$ SSSR, ot načala do kul'minacii, 1938-1953 : Dokumenty [L'antisémitisme d'État en URSS, du début à l'apogée, 1938-1953 : documents], M. : Meždunarodnyj fond 'Demokratija', 2005.

Boris Czerny

Université Caen-Normandie

$\begin{array}{llllll}\text { V } & \text { A } & \text { R } & \text { I } & \text { A }\end{array}$

\section{Robert FROST}

\section{The Oxford History of Poland-Lithuania}

Vol. I : "The making of The Polish-Lithuanian Union, 1385-1569"

Oxford : Oxford University Press, 2015, 564 p.

Voici le premier volume d'une synthèse qui n'a son pareil dans aucune langue occidentale. Un ouvrage très dense qui retrace avec soin l'évolution, pleine d'avancées et de reculs, de deux entités politiques différentes vers une union plusieurs fois proclamée et jamais parfaitement établie entre les $\mathrm{XIV}^{\mathrm{e}}$ et $\mathrm{XVI}^{\mathrm{e}}$ siècles. Non pas une histoire nationale (qui n'aurait pas de sens en parlant de cette époque), mais celle de la genèse d'une union entre deux ensembles eux-mêmes composites.

R. Frost n'a pas découvert d'archives nouvelles, mais a une connaissance parfaite de toutes celles qui sont publiées et s'appuie sur un corpus impressionnant d'études éditées en anglais, allemand (le français n'a, hélas, rien d'autre à offrir que le beau livre d'Antoine Martel datant de 1938) et surtout en polonais, lituanien, biélorussien, ukrainien et russe. Il s'agit d'une vaste revue bibliographique où les polémiques entre historiens sont bien rappelées. L'auteur y introduit souvent une opinion personnelle et mène son exposé avec la sûreté que lui donne sa compétence. Cela n'exclut pas une certaine partialité qui s'affirme chaque fois qu'apparaît, dans le tableau des évolutions politiques, la montée en Pologne du pouvoir nobiliaire, présentée comme l'affirmation d'une large volonté commune et citoyenne. Le 